\title{
Morphological assessment raises the possibility of cryptic species within the Luristan newt, Neurergus kaiseri (Amphibia: Salamandridae)
}

\author{
Mansoureh Malekian ${ }^{1}$, Hadi Khoshnamvand ${ }^{1} \&$ Yazdan Keivany $^{1}$ \\ 1. Department of Natural Resources, Isfahan University of Technology, 8415683111, Isfahan, IRAN
}

\begin{abstract}
One of the main challenges in the conservation of biodiversity is to overcome inadequate knowledge about species and their intra-specific diversity. In the present study, we attempted to assess morphological distinction of the two previously identified genetic clades within the Luristan newt (Neurergus kaiseri, Schmidt 1952) endemic to Iran, which is essential for its conservation planning. Signals of the morphological variation in $N$. kaiseri were evaluated using landmark-based geometric morphometrics of body shape and characters of osteological structures. Morphological approaches revealed consistent groupings within the species, confirming the presence of two distinct lineages (previously named as the northern and southern clades). The morphological and genetic data provide evidence for the possible co-existence of two species in $N$. kaiseri and we recommend assigning the newly recognised forms to the species level.
\end{abstract}

Keywords: geometric morphometrics, Kaiser's spotted newt, intra-specific diversity, osteology, Zagros Mountains

\section{INTRODUCTION}

$M$ orphological structures have been used, in the past 250 years, in various organisms to identify species, distinguish closely related taxa and demonstrate morphological variation among populations. Two types of morphometric techniques are commonly used, including traditional and geometric morphometrics. Geometric morphometric methods are widely used nowadays and are particularly useful in detecting morphological differences below the species level (Adams et al., 2004; Loy, 1996). Phenotypic differentiations at species and even subspecies level can be evaluated using landmark-based geometric morphometrics with a high discriminatory power (Bookstein, 1997; Nolte \& Sheets, 2005; Rohlf \& Marcus, 1993). Landmark-based geometric morphometric analysis allows visualising shape differences through thin-plate spline diagrams that can be used to portray the morphological evolution of a given lineage or group (Catalano et al., 2010).

Amphibians are in decline worldwide, yet there is a great amount of undiscovered diversity that calls for scientific explorations. The Luristan newt (Neurergus kaiseri, Schmidt, 1952) belongs to Salamandridae and is a vulnerable species, endemic to the southern Zagros Mountains of Iran (IUCN, 2018). It is highly dependent on ponds and streams and it is patchily distributed in mountainous areas. Developing conservation strategies is of high priority, however, uncertainty in its taxonomic status and intra-specific diversity hamper effective management. Illegal collection of the newt for the pet trade is one of the major factors threatening the survival of its wild populations (IUCN, 2018). When the smuggled newts are retrieved from illegal hunters, returning them to their habitat is challenging and may cause the loss of individuals due to different climatic conditions throughout its range. If the released individual survives, there is a concern about the loss of genetic diversity due to genetic erosion (Frankham et al., 2004).

Limited knowledge is currently available on $N$. kaiseri including some aspects of its ecology such as its distribution (Mobaraki et al., 2014; Sharifi et al., 2013), and demography such as age structure, longevity and growth patterns (Farasat \& Sharifi, 2015). Genetic studies based on the variation of mitochondrial control region (Farasat et al., 2016) and nuclear DNA (Rancilhac et al., 2019) revealed the presence of two genetically divergent clades (northern and southern clades) and a high geographical structuring within the species. Populations of the two clades have been isolated for about 1.5 million years and their habitats differ in temperature and precipitation (Farasat et al., 2016). Rancilhac et al. (2019) suggested that the two main clades of $N$. kaiseri could be considered distinct species, which is not currently reflected in the species taxonomy. This study did not lead to satisfactory conclusions about taxonomic 


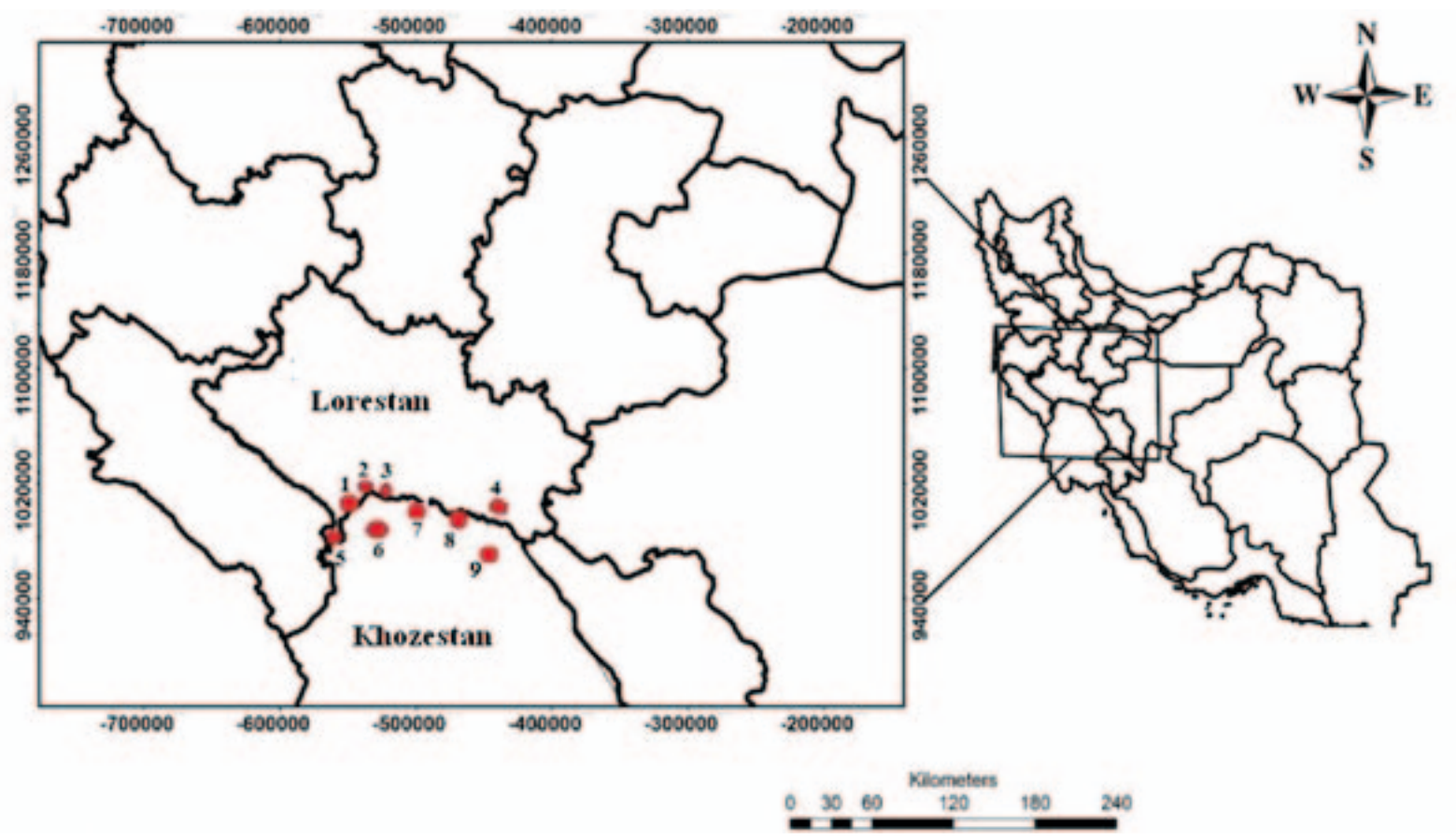

Figure 1. Sampling localities of $N$. kaiseri populations in the south-western Iran. Site names are given in Table 1.

status of the two clades because simulation studies have shown that the multiple species coalescent models do not necessarily infer true species boundaries, but might rather reflect population structure (Sukumaran \& Knowles, 2017). The external morphological characters of specimens and skeleton structure of the species are yet to be described, which can provide additional data to further support the possibility of the recognised clades (northern and southern clades) as two species. We tested the delimitation of these clades by using landmark-based geometric morphometrics of larvae and adults. We also used characters of osteological structures to compare morphological variations in the skeleton of $N$. kaiseri. The information generated from this investigation may help clarify the identity of the species, and be useful for developing conservation and management programmes.

\section{MATERIAL AND METHODS}

\section{Study area and sampling}

The distribution of $N$. kaiseri in southern Zagros Mountains of Iran (Fig. 1) encompasses a minimum convex polygon of $900 \mathrm{~km}^{2}$ between Lorestan and Khozestan Provinces (Mobaraki et al., 2014). Visual surveys for larvae or adults of the Luristan newt were conducted in the region in spring and autumn 2015 (see below). Due to the drought during the past few years, many of the ponds and springs in the region were dried out, limiting access to some of the known breeding sites (Farasat et al., 2016). However, we were able to collect samples from nine sites, covering both northern (Kolchap, Kerser, Daregoand, Vogenab) and southern (Sh.Ahmad, Tove, Bozorgab, Talezang and Mongare) populations (Table 1). Sampling sites are mainly located at high elevations and separated from each other by steep and rocky mountains.

For morphometric analyses, individuals were captured using a dip net (ring diameter of $40 \mathrm{~cm}$ ) and photographed alive without anaesthesia using a Panasonic digital camera (8 MP) (Mitteroecker \& Gunz, 2009). Larvae resemble miniature adults, differing in size, coloration and the presence of external gills, and usually reach maturity after three to four years (Consideration of Proposals For Amendment of Appendices I and II, 2010). To minimise interference and possible harm to animals, adults were sampled after the breeding season in late spring 2015. Sampling of larvae was carried out during one week in November 2015, when larvae were well developed. Individuals at the same larval development stage were selected for analyses (Alarcon-Rios et al., 2017). We were able to photograph a total of 90 adults and 112 larvae (Table 1) and all individuals were then released at the place of sampling.

The permit for measuring live $N$. kaiseri was issued by the Iranian Department of Environment. However, the vulnerable (VU) status of the species entails sampling restrictions for capturing and killing live individuals. Therefore, only deceased carcasses of $N$. kaiseri could be taken and used for osteological analyses. As the Luristan newt habitats are mainly streams, waterfalls and springs, located at high elevations (800 to $1500 \mathrm{~m}$ a.s.l) with relatively high water flow which could wash away the possible carcasses, $2 \mathrm{~mm}$ fish nets were installed in down streams and regularly checked. Dead individuals were captured by the fish net (Table1) and adults with the body length of $\sim 130 \mathrm{~mm}$ were used for the analyses. We selected similar sized specimens to minimise differences in ossification due to age. It has been suggested that body length is linearly correlated with age in many salamandrid species until maximum length is reached (Lima et al., 2000; Üzüm, 2009). Collected specimens were preserved in $96 \%$ ethanol prior to processing in the lab. 


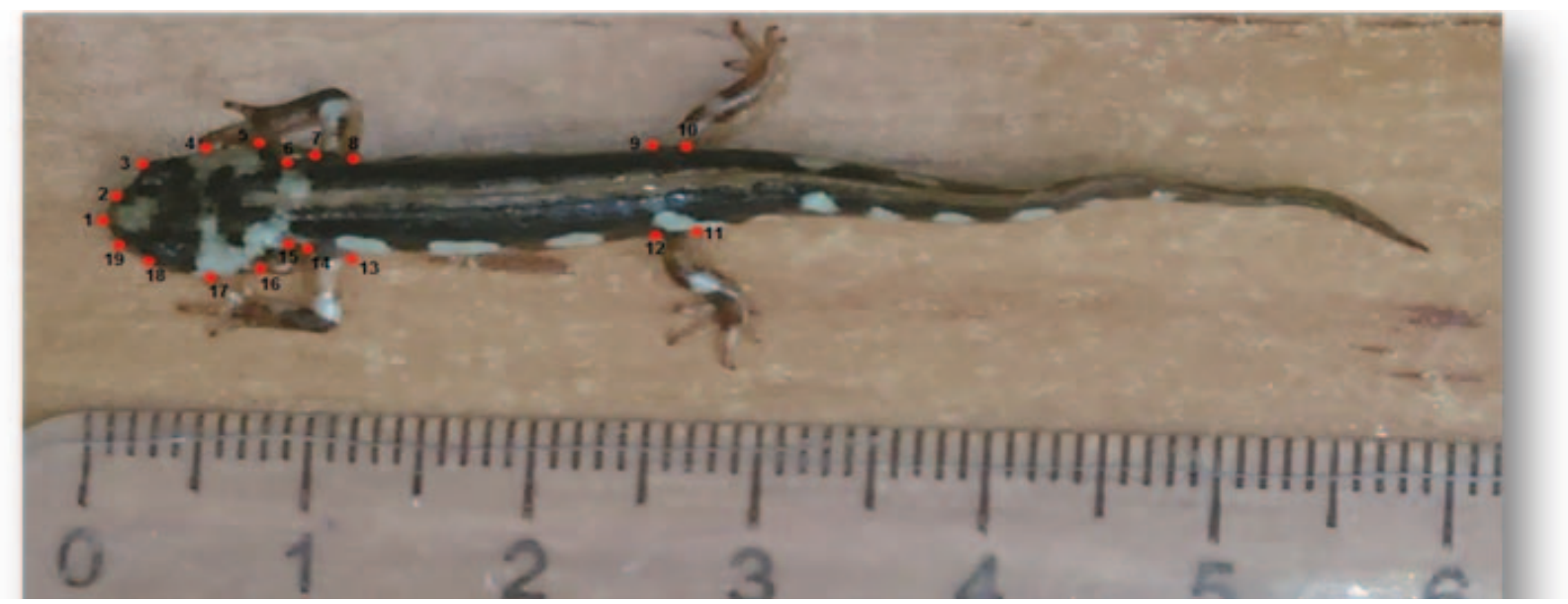

Figure 2. The defined bilaterally symmetric landmarks for extracting the shape data of N. kaiseri. 1. snout tip; 2. the beginning of the eye socket; 3 . dorsal edge of the head perpendicular to the center of eye; 4 . The bottom of the eye socket, 5 . upper edge of the jaw; 6 . bottom of the head; 7. tip of hand frame; 8 . bottom of the hand frame; 9 . tip of leg frame; 10. bottom of leg frame; 11-19. same as 10-2 (left side).

Table 1. Sampling sites and the number of samples obtained for geometric morphometrics (adults and larvae) and osteological structure of $N$. kaiseri in south-western Iran.

\begin{tabular}{|c|c|c|c|c|c|c|}
\hline \multirow[t]{3}{*}{ Site name } & \multirow[t]{3}{*}{ ID } & \multirow[t]{3}{*}{ Altitude (m) } & \multirow[t]{3}{*}{ Geographic location } & \multicolumn{3}{|c|}{ No. samples } \\
\hline & & & & \multicolumn{2}{|c|}{ Geometric morphometrics } & \multirow[t]{2}{*}{ Osteology } \\
\hline & & & & Adults & Larvae & \\
\hline Kolchap & 1 & 851 & $32^{\circ} 55^{\prime} \mathrm{N}, 48^{\circ} 22^{\prime} \mathrm{E}$ & - & 14 & 1 \\
\hline Daregol & 2 & 1050 & $32^{\circ} 55^{\prime} \mathrm{N}, 48^{\circ} 10^{\prime} \mathrm{E}$ & 15 & 14 & - \\
\hline Kerser & 3 & 1100 & $32^{\circ} 06^{\prime} \mathrm{N}, 48^{\circ} 01^{\prime} \mathrm{E}$ & 13 & 15 & 1 \\
\hline Vogenab & 4 & 950 & $33^{\circ} 00^{\prime} \mathrm{N}, 48^{\circ} 4^{\prime} \mathrm{E}$ & 15 & 12 & - \\
\hline Sh. Ahmad & 5 & 890 & $32^{\circ} 58^{\prime} \mathrm{N}, 48^{\circ} 22^{\prime} \mathrm{E}$ & - & 14 & - \\
\hline Tove & 6 & 940 & $32^{\circ} 6^{\prime} \mathrm{N}, 48^{\circ} 53^{\prime} \mathrm{E}$ & 12 & 14 & 1 \\
\hline Bozorgab & 7 & 1080 & $49^{\circ} 40^{\prime} \mathrm{N}, 48^{\circ} 00^{\prime} \mathrm{E}$ & 16 & - & - \\
\hline Talezang & 8 & 980 & $32^{\circ} 45^{\prime} \mathrm{N}, 48^{\circ} 50^{\prime} \mathrm{E}$ & - & 15 & 1 \\
\hline Mongare & 9 & 960 & $32^{\circ} 00^{\prime} \mathrm{N}, 48^{\circ} 02^{\prime} \mathrm{E}$ & 19 & 14 & 1 \\
\hline
\end{tabular}

\section{Geometric morphometrics of body shape}

Nineteen bilaterally symmetric landmarks on twodimensional images were selected on both larvae and adults of $N$. kaiseri (Fig. 2) using TpsDig2.04 (Rohlf, 2005). These landmarks included: snout tip; the beginning of the eye socket; dorsal edge of the head perpendicular to the centre of eye; the bottom of the eye socket; upper edge of the jaw; bottom of the head; tip of hand frame; bottom of the hand frame; tip of leg frame; bottom of leg frame. We followed landmark-based geometric morphometric methods (Rohlf \& Marcus, 1993) to define landmarks that are widely used and best represent the head and body morphology (e.g. Adams et al., 2007; Ivanović et al., 2013; Ivanovic \& Arntzen, 2014; Ivanović et al., 2009).

In order to minimise observer-induced errors, one person digitised the landmarks of individuals. Landmarks were then analysed using generalised Procrustes analysis (GPA, Rohlf and Slice (1990), which normalises shape data (annotated by landmarks) at equal scale, allowing for an accurate comparison of shapes regardless of their size. In order to properly evaluate whether two clades differ in their morphology, we assessed size, shape and allometric differences among clades. The snout-vent length (SVL) of all individuals were obtained and a t-test was performed for differences. The relationship between size (independent variable) and shape (dependent variable) in the two clades (northern and southern) was tested by performing a multivariate regression of shape on size (averaged by clade). Statistical significance of regressions was assessed through permutation tests with 10,000 iterations under the null hypothesis of independence between size and shape. A Procrustes ANOVA was performed, looking for a significant value in the interaction term of size and clade to identify different allometric patterns. Principal components analysis (PCA) and canonical variates analysis (CVA) were applied on shape data. All analyses were performed using PAST (Hammer et al., 2001) and MorphoJ (Klingenberg, 2011).

\section{Skeletal structure}

The deceased carcasses of $N$. kaiseri (Table 1 ) were used for osteological analyses. To clean and stain bones, the following procedure was followed (Taylor \& Van Dyke, 1985; Torres \& Ramos, 2016). First, carcasses were fixed in $500 \mathrm{ml}$ of $10 \%$ neutral formalin for 4 days and washed thoroughly under running tap water for at least one hour to remove excess formalin. Second, specimens 


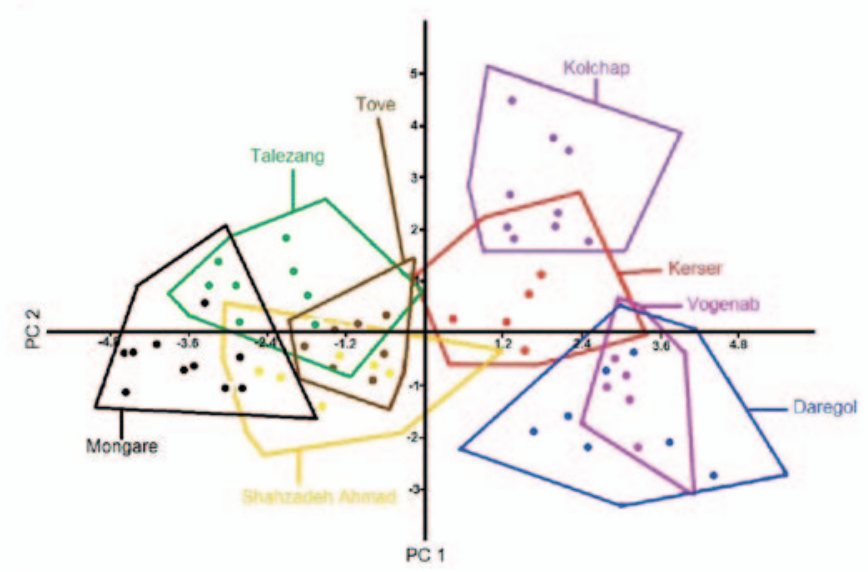

B)

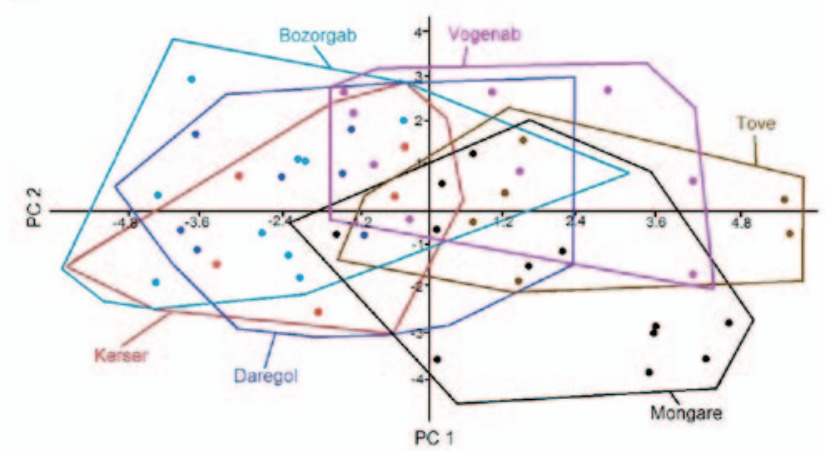

Figure 3. Scatter plots of the first two principal components with convex polygons of all larvae (A) and adults (B) of $N$. kaiseri populations in the south western Zagros Range, Iran.

Table 2. Osteological characters measured on skulls of N. kaiseri. Bone name, abbreviation, mean and standard deviation (SD) of each character are given for the northern and southern clades.

\begin{tabular}{lccc}
\hline Bone name & Abbreviation & $\begin{array}{c}\text { Northern } \\
\text { clade }\end{array}$ & $\begin{array}{c}\text { Southern } \\
\text { clade }\end{array}$ \\
\hline Atlas & At & $2.82 \pm 0.02$ & $3.21 \pm 0.01$ \\
Maxilla & Max & $9.92 \pm 0.03$ & $10.03 \pm 0.02$ \\
Premaxilla & Pm & $3.01 \pm 0.01$ & $3.12 \pm 0.01$ \\
Nasal & Nas & $2.95 \pm 0.02$ & $2.99 \pm 0.01$ \\
Prefrontal & Prfl & $2.39 \pm 0.03$ & $2.53 \pm 0.01$ \\
Frontal & Fr & $4.95 \pm 0.03$ & $5.22 \pm 0.01$ \\
Parietal & Par & $4.88 \pm 0.01$ & $5.00 \pm 0.01$ \\
Squamosal & Sq & $2.79 \pm 0.01$ & $2.85 \pm 0.01$ \\
Prootic & Pro & $4.12 \pm 0.02$ & $4.03 \pm 0.02$ \\
Otic & Otic & $3.02 \pm 0.03$ & $3.11 \pm 0.01$ \\
Cavum internasale & Ca.in & $2.20 \pm 0.01$ & $1.92 \pm 0.01$ \\
Exoccipital & Exoc & $1.07 \pm 0.03$ & $1.02 \pm 0.01$ \\
Lacrimal & L & $2.06 \pm 0.02$ & $2.10 \pm 0.02$ \\
Processus pterygoid & Pt & $3.22 \pm 0.03$ & $3.08 \pm 0.01$ \\
Parasphenoid & Prsph & $8.98 \pm 0.01$ & $9.12 \pm 0.01$ \\
Orbitosphenoid & Osph & $8.96 \pm 0.02$ & $9.15 \pm 0.02$ \\
\hline
\end{tabular}

were placed in $15 \mathrm{ml}$ of $1 \%$ alizarin red solution, holding the specimens for three days. Third, the specimens were transferred into alkaline blue stain solution for two days. Fourth, specimens were transferred into trypsin solution at $\sim 37{ }^{\circ} \mathrm{C}$, the enzyme solution was changed and the specimens were washed in distilled water every three days to avoid bacterial digestion. The stained specimens were examined using a stereomicroscope (HP SNP 120), and different skeletal elements were dissected and scanned in lateral, dorsal and ventral view, using a scanner (HP Scanjet G4050). For each specimen or view, a series of images were taken to produce a single image with maximum depth of field. The final image was drawn using CorelDrawX7 software and digitised using the TpsDig2.04 (Rohlf, 2005). The terminology of the skeletal elements follows Vassilieva et al. (2015). For comparisons, the mean of each variable was recorded for the northern and southern clades (Table 2), however, the low number of specimens precluded further quantitative comparative osteological analysis on size and allometric trends.

\section{RESULTS}

\section{Geometric morphometrics of body shape}

The results of t-tests evaluating differences in SVL showed a significant difference between the two clades in both adults and larvae. In adults, the SVL of the northern populations averaged $72.5 \pm 1.34 \mathrm{~mm}$ and in the southern clade these averaged $61.19 \pm 0.55 \mathrm{~mm}(P$ $=0.001$ ). In larvae, the SVL of the northern populations averaged $61.5 \pm 0.54 \mathrm{~mm}$ and in the southern clade this averaged $52.2 \pm 0.45 \mathrm{~mm}(P=0.004)$.

A PCA conducted on both adults and larvae showed that the sampled populations are separated into two 
A)

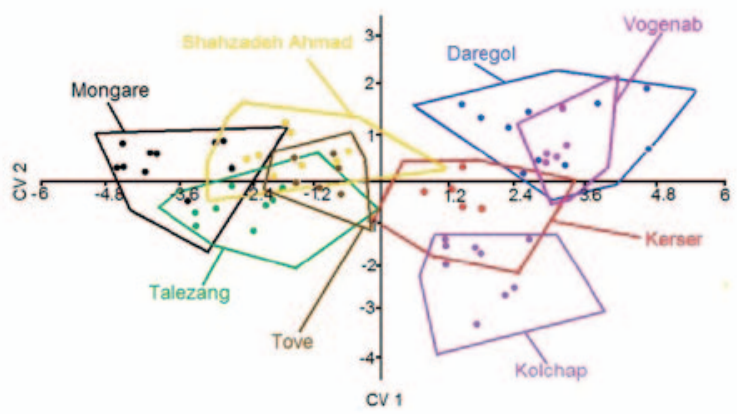

B)

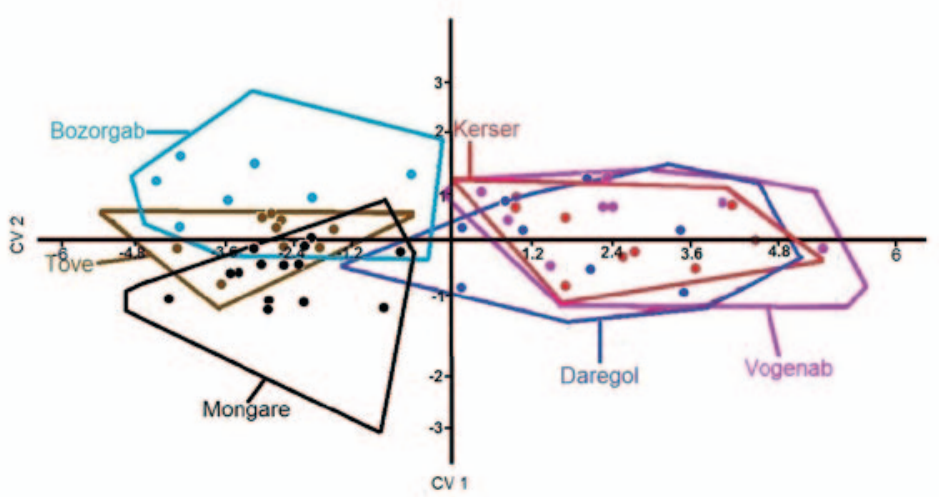

Figure 4. Scatterplots of the two first canonical axis resulted from canonical variate analysis (CVA), showing the distribution of all larvae (A) and adults (B) of N. kaiseri populations in the south western Zagros Range, Iran.

groups, corresponding to the two genetic clades (Fig. 3AB). In the PCA of adults, the first two principal components (PC1 and PC2) accounted for $78 \%$ of the total variance. In larvae, however, the first four PC accounted for a total of about $97 \%$ of the variance. The scatter plot for the

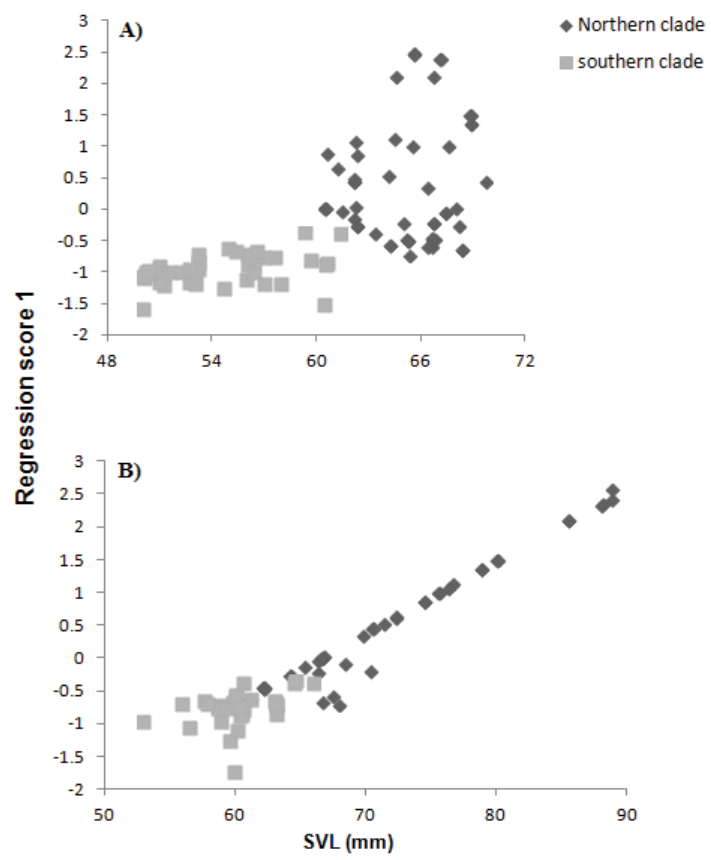

Figure 5. Multivariate regression scores of body shape variation by snout-vent length (SVL) for larvae (A) and adults (B) of $N$. kaiseri populations in the south western Zagros Range, Iran. first two principal components with convex polygons for larvae revealed two distinct groups including the northern and southern populations of $N$. kaiseri (Fig. $3 \mathrm{~A})$, though small overlaps were observed in the convex polygons of adults (Fig. 3B). The CVA further confirmed the distinction of the northern and southern clades with significant differences ( $P<0.01$, Fig. 4A-B). The Procrustes ANOVA analyses revealed significant differences in adults ( $\mathrm{F}=8.75, P=0.0001)$ and larvae $(\mathrm{F}=6.81, P=0.0009)$ between the two clades, suggesting that they have different allometric patterns. Multivariate regressions also returned significant values for both adults $(r=0.97$, $P<0.000)$ and larvae $(R=0.94, P<0.0001)$ after 10,000 permutations (Fig. 5A-B).

\section{Skeletal structure}

Overall, the skull of $N$. kaiseri showed a dense structure with severely ossified elements. Low amounts of cartilaginous elements were seen in mobile facets. Similarities were observed in the axial skeleton of both specimens. The vertebral number of each examined specimen was 50 and no variation was observed, however the number of specimens examined here was limited.

Arntzen et al. (2015) also found that in some groups such as Neurergus and Lyciasalamandra, the number of trunk vertebrae is stable, while in many groups it is subject to change. The vertebral column comprises two cervical, 16 abdominal and 32 caudal parts. Other similar components were hands and feet, which consisted of four fingers, having three (33\%, two specimens) or four phalanxes (67\%, four specimens). Seven bones 
A)

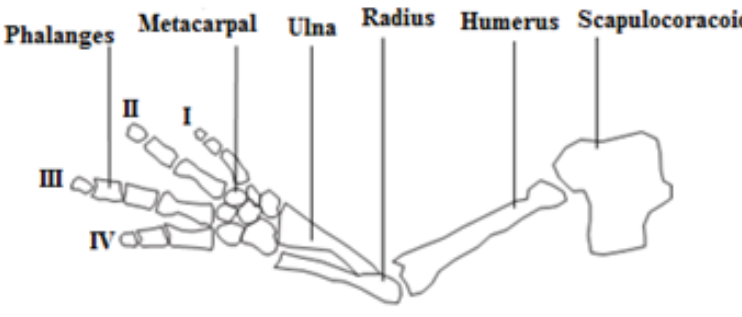

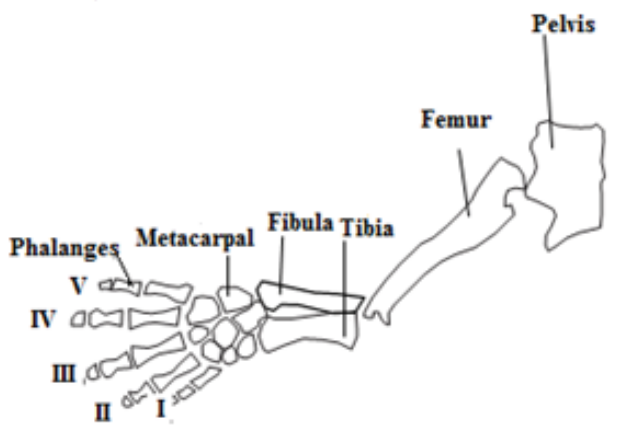

Figure 6. Hand (A) and leg (B) of N. kaiseri. I-IV: digits I-IV. Scale bar: $1 \mathrm{~mm}$.

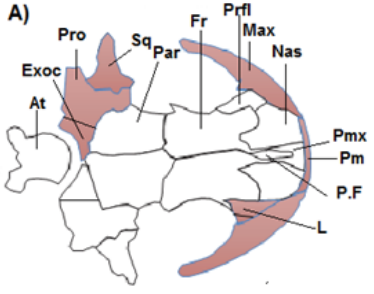

C)
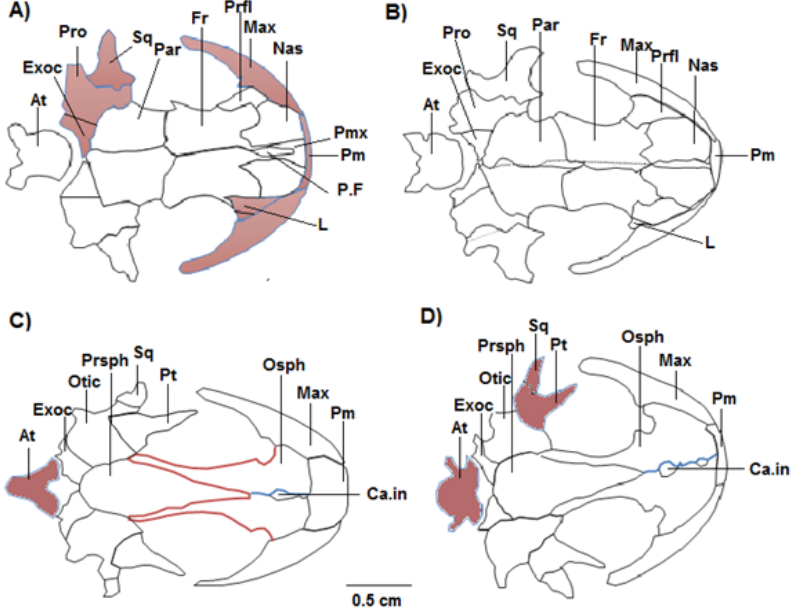

Figure 7. Osteological structures of $N$. kaiseri. Dorsal view of the skull, the northern (A) and southern (B) clade. Ventral view of the skull of the northern $(\mathbf{C})$ and southern (D) clade of N. kaiseri. Abbreviations: At: Atlas; Ca.in: Cavum internasale; Fr: Frontal; L: Lacrimal; Max: Maxilla; Nas: Nasal; P.F: Premaxilla Foramen; Pm: Premaxilla; Par: Parietal; Prfl: Prefrontal; Pro: Prootic; Sq: Squamosal; Exoc: Exoccipital; Otic: Otic; Osph: Orbitosphenoid; Prsph: Parasphenoid; Pt: Processus Pterygoid.

are observed in the metacarpal and the number of metatarsus bones is eight (Fig. 6A-B).

Comparing the skull elements revealed some clear size (Table 2) and shape (Fig. 7A-D) differences between the northern and southern clades. For example, in dorsal views, shorter maxilla bones are seen in the northern clade compared to those in the southern (Table 2). Squamosal bones were completely different in size and shape in both specimens. Exoccipital bones are seen in the southern clade as separate bones, connected to the pterygoids bones and then to the occipitals. In the ventral view, the squamosal bones of the southern clade have longer parts compared to the northern (Table 2). The Atlas vertebra has two transverse processes in the southern clade. The premaxillary bone is bigger in the southern clade (3.12 \pm 0.01 ) compared to the northern $(3.01 \pm 0.01)$. Prefrontal, frontal, parietal, otic, parasphenoid and orbitosphenoid were other larger skull elements in the southern clade.

The nasal bone is wide and elongated in the length of the skull roof in both clades. The vomer bone makes a pit between the nasal near the premaxilla, which is wider in the northern clade than the southern. The internasale cavity is larger in the northern clade $(2.20 \pm 0.01 \mathrm{~mm})$ compared to the southern specimen $(1.92 \pm 0.01 \mathrm{~mm})$ (Table 2). In addition, prootic, exoccipital and processus pterygoid were larger in the northern clade compared to the southern one (Table 2).

\section{DISCUSSION}

Based on the data presented here, there is significant morphological diversity within N. kaiseri. Our approaches (landmark-based geometric morphometrics of body shape and characters of osteological structures) revealed consistent groupings within the species, confirming the distinction of the two previously identified genetic clades (the northern and southern clades, Farasat et al., 2016; Rancilhac et al., 2019). Ecological differences also exist in the habitats of the two clades. The northern populations are located at higher elevations in a more humid climate than the southern clade. Habitat suitability models showed that northern populations occupy habitats that are cooler with lower winter temperatures and higher summer rainfall (Farasat et al., 2016). In contrast, southern populations are characterised by warmer habitats with higher winter temperatures and wetter winters (Farasat et al., 2016). The average elevation and average annual precipitation received by the northern and southern clades are $1050 \mathrm{~m}$ a. s. I. and $670 \mathrm{~mm}$, and $870 \mathrm{~m}$ a. s. I. and $450 \mathrm{~mm}$, respectively. In addition, based on the field surveys, habitats of the southern clade are mainly streams with fast-flowing water, while habitats are mostly ponds and springs with standing water in the northern clade. The morphological patterns of the southern clade may be an adaptation to lentic or lotic habitats to increase their ability to swim against the water flow. Differences in body morphology associated with an increase in maximising swimming speed is suggested for the European newts of the genus Triturus (Gvozdik \& Van Damme, 2006). Research has shown that morphological differences may be attributed to environmental factors or genetic differences during the developmental processes (Van Buskirk, 2002, 2009), indicative of features that enable newts to live in different 
aquatic ecosystems (Hopkins, 2007). Specific features of these newts, such as possible low dispersal abilities, may have also contributed to the intra-specific diversity of $N$. kaiseri. The results of this study provide adequate evidence for the potential co-existence of two species in the distribution range of $N$. kaiseri in south western Iran. Therefore, a taxonomic revision of $N$. kaiseri is recommended.

Due to the vulnerability of the species and sampling restrictions, we were only allowed to collect deceased individuals to perform the osteological analysis. The skull of $N$. kaiseri has a dense structure and is severely ossified. In salamanders that live in mountainous habitats (usually at altitudes above $900 \mathrm{~m}$ ), skulls tend to be harder and stronger compared to those that inhabit the flatter areas (Buckley et al., 2010). The Luristan newt habitats are mainly streams and springs located at high elevations (800 to $1500 \mathrm{~m}$ a.s.l), separated from each other by steep and rocky mountains. Considering the habitats of $N$. kaiseri with high altitudes, a high degree of ossification may be expected.

Despite the small sample size, differences in the skull skeleton were observed between the two clades. Some of the skull elements were clearly larger or wider in the northern clade, such as internasale cavity. The size of the internasale cavities may be an adaptation to living in higher altitudes with lower oxygen pressures. Osteological features of $N$. kaiseri in both specimens may reflect changes in its composing structures with adaptations to the habitat and can be described as evolutionary process that gradually accumulated as modified anatomical structures. Amphibians living in high elevations tend to have lower metabolic rates, larger body sizes and greater longevity than their low altitude relatives (Morrison \& Hero, 2003). Such variations have been attributed to low environmental temperatures and the short period of annual activity (Wells, 2007).

Our findings have important conservation implications. The Luristan newt is listed on the Appendix I of the Convention on International Trade in Endangered Species of Wild Fauna and Flora (CITES, 2010), however, infrequent illegal collection of the newt for the pet trade threatens the survival of its wild populations. Releasing retrieved newts from illegal hunters into habitats should be conducted with adequate caution due to significant genetic (Farasat et al., 2016; Rancilhac et al., 2019), morphological (this study), and ecological differences (Farasat et al., 2016; Sharifi et al., 2013; Steinfartz et al., 2002) between the northern and southern clades. Released individuals may not survive in the wild and if they do, there is a concern about the loss of diversity due to genetic erosion or out-breeding depression (Frankham et al., 2004). Our findings can assist regional environmental managers to take informed decisions, preventing introgressions between the two clades.

\section{CONFLICT OF INTEREST}

We wish to confirm that there are no known conflicts of interest associated with this manuscript and there has been no significant financial support for this research.

\section{REFERENCES}

Adams, D. C., Rohlf, F. J., \& Slice, D. E., (2004). Geometric morphometrics: Ten years of progress following the revolution. Italian Journal of Zoology 71, 5-16.

Adams, D. C., West, M. E., \& Collyer, M. L., (2007). Locationspecific sympatric morphological divergence as a possible response to species interactions in West Virginia Plethodon salamander communities. Journal of Animal Ecology 76, 289-295. doi: 10.1111/j.1365-2656.2007.01210.x

Alarcon-Rios, L., Velo-Anton, G., \& Kaliontzopoulou, A., (2017). A non-invasive geometric morphometrics method for exploring variation in dorsal head shape in urodeles: sexual dimorphism and geographic variation in Salamandra salamandra. Journal of Morphology 278, 475-485. doi: 10.1002/jmor.20643

Arntzen, J. W., Beukema, W., Galis, F., \& Ivanović, A., (2015). Vertebral number is highly evolvable in salamanders and newts (family Salamandridae) and variably associated with climatic parameters. Contributions to Zoology 84, 85-113.

Bookstein, F. L., (1997). Morphometric Tools for Landmark Data: Geometry and Biology. Cambridge, USA, Cambridge University Press. $459 \mathrm{pp}$.

Buckley, D., Wake, M., \& Wake, D., (2010). Comparative skull osteology of Karsenia koreana (Amphibia, Caudata, Plethodontidae). Journal of Morphology 271, 533-558.

Catalano, S. A., Goloboff, P. A., \& Giannini, N. P., (2010). Phylogenetic morphometrics (I): the use of landmark data in a phylogenetic framework. Cladistics 26, 539 - 549.

CITES. (2010). Convention on International Trade in Endangered Species of Wild Fauna and Flora, Considerations for Proposals of Amendment of Appendices I and II, CoP15 Prop.14, Doha, Qatar. http://www.cites.org/eng/cop/15/ prop/E-15\%20Prop-14.pdf (accessed 2.4.16).

Consideration of Proposals For Amendment of Appendices I and II. 2010). Convention on International Trade in Endangered Species of Wild Fauna and Flora, CoP15 Prop. 14: 1-10. Accessed August 10, 2018 at http://www.cites. org/eng/cop/15/prop/E-15-Prop-14.pdf.

Farasat, H., Akmali, V., \& Sharifi, M., (2016). Population genetic structure of the endangered Kaiser's mountain newt, Neurergus kaiseri (Amphibia: Salamandridae). Plos One 11, e0149596.

Farasat, H., \& Sharifi, M., (2015). Ageing and growth of the endangered Kaiser's mountain newt, Neurergus kaiseri (Caudata: Salamandridae), in the southern Zagros range, Iran. Journal of Herpetology 50, 120-125.

Frankham, R., Ballou, D., \& Briscoe, D. A., (2004). A Primer of Conservation Genetics, Cambridge University Press, Cambridge.

Gvozdik, L., \& Van Damme, R., (2006). Triturus newts defy the running-swimming dilemma. Evolution 60, 2110-2121.

Hammer, Ø., Harper, D. A. T., \& Ryan, P. D., (2001). PAST: Paleontological statistics software package for education and data analysis. Palaeontologia Electronica 4, 1-9.

Hopkins, W. A., (2007). Amphibians as models for studying environmental change. ILAR Journal 48, 270-277. doi: 10.1093/ilar.48.3.270

IUCN. (2018). The IUCN Red List of Threatened Species. Version 2018-1. available at www.iucnredlist.org. accessed on 17 July 2018. 